\title{
Análisis de la integración del iPad en el aula desde la perspectiva del alumno: proyecto piloto de la UCJC
}

\author{
Jorge GallaRdo CAMACHO \\ Universidad Camilo José Cela \\ jgallardo@ucjc.edu
}

\begin{abstract}
Resumen
Este artículo analiza el uso de la tableta digital iPad en el aula en el marco del proyecto piloto de la Universidad Camilo José Cela (UCJC). La investigación refleja la experiencia de los alumnos después del primer año de uso de la tableta digital. El estudio analiza la utilización del iPad como herramienta académica a través de 35 encuestas. Entre las conclusiones, destaca la percepción positiva del alumnado por la integración de esta nueva tecnología educativa en el aula. Los estudiantes reconocen que mejoran sus habilidades profesionales y expositivas para ser más competitivos en el mercado laboral de la Comunicación.
\end{abstract}

Palabras clave: Tableta digital, iPad, TIC, nuevas tecnologías, educomunicación.

\section{Analysis of the integration of the iPad in the classroom from the student perspective: UCJC pilot project.}

\begin{abstract}
This paper analyzes the use of digital tablet iPad in the classroom as part of the pilot project at the University Camilo Jose Cela (UCJC). The research reflects the experience of students after the first year of using digital tablets. The study analyzes the use of iPad as an academic tool through 35 surveys. Among the conclusions, we note the student's positive perception about the integration of this new educational technology in the classroom. Finally, students recognize the improvement of their professional and presentation skills to be more competitive in the labor market of Communication.
\end{abstract}

Key words: Digital tablet, iPad, ICT, new technologies, educommunication

\section{Referencia normalizada:}

Gallardo Camacho, J. (2013) Análisis de la integración del iPad en el aula desde la perspectiva del alumno: proyecto piloto de la UCJC. Historia y Comunicación Social. Vol. 18 No Especial Octubre. Págs. 399-410.

Sumario: 1. Introducción. 1.1. Estado de la cuestión. 1.2. La tableta digital: la pantalla que más crece. 1.3. El proyecto piloto de la UCJC. 2. Metodología. 3. Resultados. 3.1. La experiencia personal del alumno. 3.2. La percepción del uso docente de la tableta digital. 4. Conclusiones. 5. Bibliografía.. 


\section{Introducción}

En una sociedad cada vez más tecnológica es necesario analizar cómo las Tecnologías de la Información y Comunicación (TIC) modifican las aulas universitarias. En esta investigación nos vamos a centrar en el uso de la tableta digital y, concretamente, del iPad en el contexto del proyecto piloto de la Facultad de Comunicación de la Universidad Camilo José Cela (Madrid). Ante la falta de investigaciones que analicen el uso de las tabletas digitales en el aula, el objetivo de este artículo es el de compartir con los docentes del Área de Comunicación la experiencia de la tableta digital en el ámbito académico desde la perspectiva de los alumnos.

\subsection{Estado de la cuestión}

Existen pocos estudios sobre la integración del iPad en el aula. Pero, mayoritariamente, las investigaciones existentes concluyen que la presencia de esta herramienta tiene más aspectos positivos que negativos. Por ejemplo, el informe de Webb (2012) analiza las respuestas de 310 estudiantes de un instituto de Reino Unido de entre siete y trece años, y confirma que casi nueve de cada diez estudiantes están felices por utilizar el iPad en el aula. En lo que se refiere al uso que dan esos alumnos a las tabletas digitales destacan tres aspectos: la investigación de contenidos en Internet $(>60 \%)$, la creación de presentaciones $(>50 \%)$ y la realización de esquemas mentales y organización del trabajo con la aplicación Popplet $(>50 \%)$.

Sin embargo, ¿es útil el uso del iPad en los estudios de Comunicación? Una investigación centrada en los alumnos de Periodismo (Viñas y Cabezuelo, 2012) coincide con los efectos positivos del iPad al asegurar que las tabletas digitales abren la posibilidad de consumir contenidos de manera no lineal; es decir, que esta tecnología ayuda al estudiante para que amplíe la información en cualquier momento. Coincide así con Esteve y Sandoval (2006) cuando aseguran que las nuevas tecnologías facilitan un acceso más libre y amplio a los datos informativos sin limitaciones espaciales y temporales. Por otro lado, es destacable el caso de la Facultad de Comunicación de la Universidad San Jorge de Zaragoza, que fue la primera que implantó la tecnología táctil entre todos sus alumnos antes de la aparición del iPad (Sierra y Cabezuelo, 2010). La experiencia ha quedado registrada en una investigación que concluye que se aplicó el uso del tablet-PC con "nuevos enfoques metodológicos y técnicas pedagógicas activas" (Vadillo y Marta, 2010). Sin embargo, es obvio que el uso de la tableta digital es más útil en la impartición de asignaturas relacionadas con la expresión gráfica y el dibujo ya que "el tamaño de la pantalla del iPad y la facilidad para realizar zoom la hace apropiada para su utilización" (Saorín et al. 2011).

Por su parte, Palma-Nieto (2012) también dice que el iPad es positivo por su característica física (ligero, fácil de usar y porque ahorra gastos en libros a medio plazo), por la modificación en la manera de aprender de los estudiantes (mejoran la calidad de las presentaciones y promueve el pensamiento crítico) y por permitir una educación más personalizada. En la misma línea, Guerra (2001) advirtió, hace más de dos 
lustros, de que el uso de la tecnología en el aula incrementaría "el aprendizaje, al forzar al alumno a involucrarse en lo que está aprendiendo".

Entre las ventajas del uso de las TIC en el aula está el incremento de la interacción. Los investigadores Vadillo y Marta (2010) concluyen que las TIC posibilitan en el aula el análisis y la reflexión (en el nivel cognoscitivo); la autoestima, productividad y profesionalización (en el nivel procesual); y la interacción y participación (en el nivel actitudinal). Como conclusión, el uso del iPad impulsa el denominado por Bartolomé y Aiello (2006) como "Blended learning": entendido como "el diseño docente en el que tecnologías de uso presencial y no presencial se combinan en orden para optimizar el proceso de aprendizaje".

El mayor estudio cuantitativo que analiza la presencia de las TIC en el aula es el encargado por la Comisión Europea con 190.000 respuestas de alumnos y profesores de países de la Unión Europea. El informe concluye que la penetración de las nuevas tecnologías en las aulas puede no haber aumentado como se esperaba y que, por eso, las políticas deben estar centradas en las aulas "tanto en la gestión eficaz del aprendizaje como en la provisión de las TIC" (European Commission, 2013: 156). Paralelamente, otro estudio que analiza la opinión de los alumnos con respecto a la docencia en el marco del EEES (Espacio Europeo de Educación Superior) destaca que los estudiantes están menos interesados por la clase magistral y buscan métodos de enseñanza menos tradicionales (Vadillo, Marta y Cabrera, 2010). Consecuentemente, para cumplir los objetivos del EEES hay que aplicar el uso de las nuevas tecnologías en el aula y superar el reto cada vez más complejo del proceso de enseñanza-aprendizaje (Sierra y Cabezuelo, 2010).

Otro de los efectos positivos del uso de la tableta digital está relacionado con la reducción de la brecha digital entre alumnos y profesores. Es decir, el docente es la figura clave para implementar el uso de esta tecnología y por ello necesita enfrentarse a una formación diversificada. Pero además, con el uso del iPad, se propone reducir la denominada "brecha de pertinencia": entendida ésta como la diferencia entre lo que necesitan los profesores y lo que ofrecen los recursos digitales (Garrido et al., 2008). Lo cierto es que la llegada del iPad modifica la guía docente y, por eso, el $55 \%$ de los docentes siente que tiene que cambiar notablemente su metodología y adaptarse (Webb, 2012).

Ante la reticencia de los profesores por adquirir competencias digitales, Pérez y Cabezuelo (2011) creen que para que se rompa la falta de confianza entre docentes y tecnología es necesario que los profesores apliquen las TIC a través de experiencias experimentales con blogs u otros recursos de Internet, independientemente de la asignatura que impartan. Por ejemplo, en el inicio de la implementación de las nuevas tecnologías, como ha pasado con el caso del Proyecto Medusa en Canarias, el profesorado ha introducido las tabletas en el aula como algo complementario sin que suponga un replanteamiento significativo del modelo didáctico empleado hasta el momento (Area, 2009). De esta manera, Silva y Collao (2013) advierten de que estos 
proyectos de innovación deben tener en cuenta un "diseño de ambientes controlados que generen la información necesaria para la aceptación final de los docentes".

\subsection{La tableta digital: la pantalla que más crece}

El Interactive Advertising Bureau (IAB, 2013) destaca que los dispositivos que más crecen son las tabletas digitales (con un 43\% de penetración en 2013 frente al $23 \%$ de 2012) y los teléfonos inteligentes (con un $21 \%$ de crecimiento en tan solo un año). Según este estudio las tabletas digitales se han convertido en una pantalla cada vez más presente en los hogares. Sin embargo, el principal uso diario que le dan sus usuarios se centra en las redes sociales (49\%) y en actividades lúdicas (46\%) ya que solo el $16 \%$ reconoce utilizarlo diariamente para leer libros o para su formación personal. No obstante, el usuario de las tabletas digitales destaca por darle un uso más académico a este dispositivo con respecto a los teléfonos inteligentes ya que las tabletas son usadas un 5\% más para fines consultivos y educativos. Con respecto al lugar de manejo de las tabletas, destacan los espacios privados y seguros como el hogar; es decir, su utilización en lugares públicos es baja con respecto al teléfono móvil. Una investigación anterior destaca que el uso de las tabletas en el centro de trabajo o de estudios es solo "muy frecuente" entre el 16\% de los usuarios (IAB, 2012).

Por otra parte, el equipamiento tecnológico se muestra estrechamente relacionado con la edad: se da mayor presencia del público más joven en el uso de portátiles, consolas, teléfonos y tabletas (IAB, 2012). En este contexto, la Unión Europea ha pedido que se aprovechen educativamente los altos niveles de uso de los teléfonos móviles privados de los alumnos ya que 8 de cada 11 estudiantes declaran utilizar habitualmente el teléfono móvil en clase con fines de aprendizaje (European Commission, 2013). Según León (2012) hemos pasado de la "generación del pulgar" a la "generación del índice". Otros investigadores van más allá y aseveran que el iPad aporta "la magia de aprender con los dedos" (Sánchez y Soro, 2012). Pero las tabletas son plataformas que trascienden a lo lúdico ya que nuestro futuro inmediato está al alcance de nuestro dedo índice en la interacción con dispositivos por lo que "cada vez será más complicado no incorporarlos al día a día de los más pequeños" (León, 2012: 180). Y, en ese día a día, la escuela, el instituto y la universidad han de adaptarse a las nuevas costumbres de interacción y comunicación.

\subsection{El proyecto piloto de la UCJC}

El proyecto piloto de la UCJC es pionero en cuanto a la implementación del iPad desde el primer curso de grado (2012-2013) hasta la finalización de los estudios (2015-2016). La UCJC ha subvencionado la adquisición del iPad a 10 profesores del primer curso de la Facultad de Comunicación (Periodismo, Comunicación Audiovisual y Relaciones Públicas y Publicidad). Las ayudas, como es lógico, también han ido dirigidas a la obtención del iPad para los alumnos de primer curso. De esta manera, el grado de penetración de la tableta digital en el aula ha crecido notable- 
mente en la Facultad como veremos. Por otra parte, la experiencia de este proyecto piloto servirá para implementarse en el resto de estudios de la universidad.

Los profesores implicados recibieron cursos de formación y firmaron un acuerdo para comprometerse a utilizar el iPad como herramienta docente. Los objetivos del proyecto reflejados en la documentación de la UCJC son:

- Mejorar los ritmos de enseñanza-aprendizaje.

- Reforzar al docente con el uso de aplicaciones más acordes a la titulación.

- Motivar al alumnado para trabajar de manera más interactiva e incrementar su atención.

- Mejorar las competencias del alumno en TIC de cara a su futuro profesional.

\section{Metodología}

La experiencia reflejada en este artículo de investigación se limita a la asignatura "Lenguaje audiovisual, escrito y publicitario" impartida por el profesor Jorge Gallardo en el primer curso de la Facultad de Comunicación de la UCJC. La asignatura pertenece al proyecto piloto del uso del iPad en el aula y tenía a 35 alumnos inscritos. Toda la docencia se impartió con el iPad en el aula (incluso el día del examen). Además, el profesor premió las presentaciones y prácticas realizadas con el iPad para potenciar su uso en clase.

La metodología utilizada para analizar la opinión de estos alumnos ha sido cuantitativa y para recabar la información se ha utilizado el procedimiento de la encuesta a través de 35 cuestionarios anónimos realizados el día del examen final (junio de 2013, fecha en la que finaliza la relación alumno-profesor en la asignatura). Los cuestionarios se han inspirado en investigaciones previas que han analizado la presencia de tabletas digitales en las aulas (Vadillo y Marta, 2010; Webb, 2012). Constan de 17 ítems. Los dos primeros relacionados con la edad y los estudios de grado del encuestado. El resto del cuestionario se divide en dos bloques: el primero, se refiere a las preguntas relacionadas con el uso del iPad que hace el alumnado en clase; el segundo, hace alusión al uso del iPad por parte del profesor en el aula. Posteriormente, los resultados fueron tabulados en Excel para su tratamiento estadístico.

De cara a la exposición de los resultados, hemos tenido en cuenta dos muestras. La primera es la muestra inicial que representa a todos los alumnos $(\mathrm{N} 1=35)$; y, la segunda, la que representa solo a los estudiantes que tienen iPad en clase (N2=27). No obstante, hemos tenido en cuenta la opinión de la muestra N1 para algunos casos ya que no hace falta poseer el iPad para valorar aspectos relacionados con su uso en clase (principalmente para valorar la utilización que el profesor ha hecho de la tableta digital). Las preguntas que puedan ser respondidas por los estudiantes, aunque no tengan un iPad, se mostrarán según N1=35. Sin embargo, hay casos en los que la pregunta conlleva la necesidad de poseer el dispositivo (en ese caso los Gráficos y 
Tablas se han hecho en función de $\mathrm{N}=27)$. La edad media de la muestra $(\mathrm{N} 1=35)$ es de 19,3 años.

Aunque los resultados no son representativos del universo de la comunidad educativa de España por tratarse de una muestra reducida, sí sirven como reflejo de una experiencia pionera concreta. De ahí el objetivo de compartir el conocimiento, las experiencias y "potenciar el intercambio de aprendizajes" como requisitos para impulsar la penetración de las TIC en el aula (Garrido et al., 2008).

\section{Resultados}

Los resultados serán expuestos en dos apartados: el primero, relacionado con la experiencia personal del alumno con el iPad en el aula; y el segundo, relacionado con la percepción del alumno sobre el uso que ha hecho el profesor del dispositivo en clase. Como hemos explicado en la metodología, tenemos la muestra principal $(\mathrm{N} 1=35)$ y la muestra específica $(\mathrm{N} 2=27)$ que representa solo a los alumnos con iPad (Tabla 1).

Tabla 1. Penetración y subvención del iPad en el aula.

\begin{tabular}{|l|l|l|}
\hline & \multicolumn{1}{|c|}{ Sí } & \multicolumn{1}{c|}{ No } \\
\hline Alumnos con iPad (N1=35) & $70,3 \%(27)$ & $29,7 \%(8)$ \\
\hline Subvención de la UCJC (N2=27) & $81,5 \%$ & $18,5 \%$ \\
\hline
\end{tabular}

Fuente: Elaboración propia.

En la Tabla 1 vemos que el $81,5 \%$ de los alumnos con iPad (N2=27) ha obtenido su dispositivo gracias a la subvención recibida por parte de la universidad. La edad de los estudiantes y la utilidad potencial del iPad como objeto deseado fuera y dentro de la escuela han ayudado a la adquisición del producto. Algo que ha facilitado el desarrollo del proyecto piloto por parte de los 10 docentes involucrados.

\subsection{La experiencia personal del alumno}

Los resultados han sido muy positivos en cuanto al uso de la tableta digital por parte de los alumnos en el aula. Principalmente, porque la mayoría de los estudiantes ha utilizado "habitualmente" o "siempre" la tableta digital (Gráfico 1). 
Gráfico 1. Frecuencia de uso del iPad en clase $(\mathrm{N} 2=27)$.

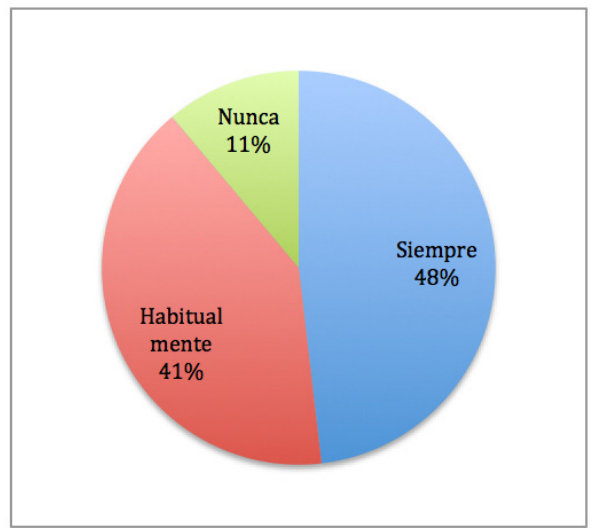

Fuente: Elaboración propia.

Por tanto, el $89 \%$ de los estudiantes con iPad reconoce utilizarlo en clase. Pero, ¿para qué?

Gráfico 2. Principales usos del iPad del alumno en clase.

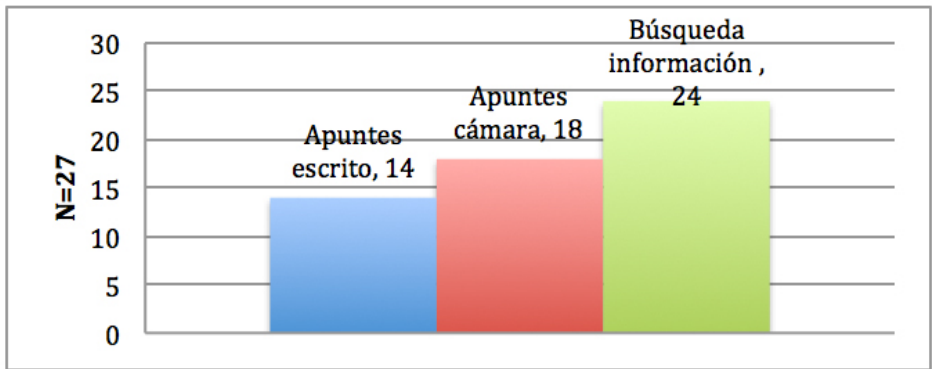

Fuente: Elaboración propia.

En el Gráfico 2 observamos que, entre los principales usos del iPad, destaca la búsqueda de información (24 de 27) coincidiendo con los resultados de otras investigaciones (Webb, 2012). Pero nos sorprenden las respuestas relacionadas con la toma de apuntes ya que hay más estudiantes que aseguran tomar fotografías (18) frente a los que escriben con el teclado táctil en su dispositivo (16). Cabe destacar que la inclusión de esta opción en los cuestionarios se debió a la observación del docente de este comportamiento por parte del alumnado.

La navegación y el uso de las tabletas digitales se realiza principalmente a través de aplicaciones (IAB, 2013). De ahí, la importancia de conocer las aplicaciones concretas utilizadas por los alumnos. 
Gráfico 3. Aplicaciones del iPad más utilizadas en clase.

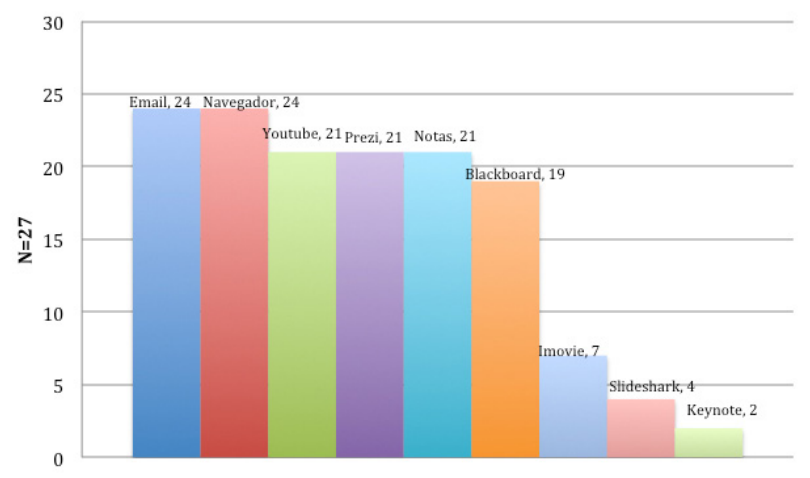

Fuente: Elaboración propia.

En el Gráfico 3 destaca el uso del correo electrónico (24) y el navegador (24). En segundo lugar, se encuentran las aplicaciones Youtube y Notas (21). Pero hay que tener en cuenta que el tipo de aplicaciones más utilizadas son las relacionadas con las exposiciones en clase: Prezi (21), Slideshark (4) y Keynote (2) son tres programas para exponer que alcanzan en conjunto una utilización del $100 \%$ de la muestra (N2=27). El motivo de esto es que el profesor premiaba a los estudiantes que exponían con iPad en el aula y, por tanto, era necesario descargar estos programas. Por otra parte, Youtube también formaba parte de una práctica en clase consistente en la realización de un canal propio en grupo. Blackboard (19) además destaca al tratarse de la plataforma virtual docente en la que pueden acceder los alumnos para descargar apuntes o ver sus notas. Por último, iMovie (7) es una aplicación sugerida por el profesor para editar vídeos en la práctica de Youtube.

\subsection{La percepción del uso docente de la tableta digital}

La percepción que los estudiantes tienen del uso que el profesor ha hecho del iPad es mayoritariamente positiva.

Tabla 3. El uso del iPad como potenciador de competencias en TIC.

\begin{tabular}{|l|c|c|c|}
\hline & Sí & No & Ns/nc \\
\hline Uso positivo del profesor del iPad para los alumnos (N1=35) & $91,4 \%$ & $5,7 \%$ & $2,9 \%$ \\
\hline $\begin{array}{l}\text { El iPad ha potenciado habilidades profesionales en } \\
\text { Comunicación de los alumnos (N1=35) }\end{array}$ & $57,1 \%$ & $34,3 \%$ & $8,6 \%$ \\
\hline $\begin{array}{l}\text { El iPad ha potenciado las habilidades expositivas del alumnado } \\
(\mathrm{N} 1=35)\end{array}$ & $74,3 \%$ & $20 \%$ & $5,7 \%$ \\
\hline Todos los profesores deberían usar el iPad en clase (N1=35) & $57,1 \%$ & $40 \%$ & $2,9 \%$ \\
\hline
\end{tabular}

Fuente: Elaboración propia. 
En la Tabla 3 vemos que el 91,4\% de los estudiantes valora como positivo el uso del iPad en el aula por parte del profesor. Pero lo más importante, la mayoría (un $57,1 \%$ ) cree que el uso de la tableta digital ha mejorado y potenciado sus habilidades como profesionales de la Comunicación. Ese porcentaje crece exponencialmente (hasta un $74,3 \%$ ) cuando se les pregunta si creen que han mejorado sus habilidades expositivas (recordemos que las aplicaciones más utilizadas son las relacionadas con las presentaciones en el aula como se indica en el Gráfico 3). Por otra parte, los alumnos estiman en su mayoría que todos los profesores deberían utilizar el iPad en clase frente a un $40 \%$ que opina lo contrario.

Pero centrémonos en la percepción que tienen los alumnos del cambio que supone la utilización de las TIC en el aula con respecto a otras herramientas docentes más tradicionales.

Tabla 4. El uso del iPad como nueva herramienta tecnológica frente a otras estrategias docentes.

\begin{tabular}{|l|c|c|c|}
\hline & Sí & No & Ns/nc \\
\hline ¿Un profesor debería prohibir el uso del iPad en clase? $(\mathrm{N}=35)$ & $2,8 \%$ & $94,4 \%$ & $2,8 \%$ \\
\hline ¿Se debería prohibir el uso del teléfono en el aula? $(\mathrm{N}=35)$ & $40 \%$ & $57,1 \%$ & $2,9 \%$ \\
\hline ¿Se debería prohibir el ordenador personal? $(\mathrm{N}=35)$ & $0 \%$ & $97,1 \%$ & $2,9 \%$ \\
\hline El iPad acabará con los libros de texto $(\mathrm{N}=35)$ & $57,1 \%$ & $40 \%$ & $2,9 \%$ \\
\hline
\end{tabular}

Fuente: Elaboración propia.

Con respecto a las preguntas relacionadas con las prohibiciones de nuevas tecnologías en el aula, los alumnos no aceptarían ningún tipo de censura (Tabla 4). Tras la experiencia de los estudiantes con el iPad no conciben la posibilidad de su prohibición y por eso solo un $2,8 \%$ comprendería su censura. El uso del ordenador en el aula parece estar más asumido si cabe y ninguno de los 35 alumnos apoyarían su prohibición. Tan solo existe algo más de disensión con respecto al uso del teléfono móvil en clase ya que un $40 \%$ de los estudiantes comprendería su prohibición. En este caso se abre el debate ya que mientras la Comisión Europea (2013) advierte de la potencialidad positiva de los teléfonos inteligentes en las aulas, es cierto que se hace complicado controlar que los alumnos hagan un uso $100 \%$ académico de su terminal (los teléfonos inteligentes conllevan usos más personales y tienen aplicaciones como Whatsapp que invitan a la distracción). En definitiva, los estudiantes son conscientes de la utilidad de las nuevas tecnologías en el aula y casi seis de cada diez creen que el iPad acabará con los libros de texto.

\section{Conclusiones}

El uso de las TIC y, concretamente de las tabletas digitales en el aula, son recibidas de manera positiva por los alumnos en el proyecto piloto de la UCJC. De hecho, en 
la Tabla 3 se encuentran las 3 preguntas del cuestionario referidas directamente a si la experiencia ha sido positiva o negativa; y los estudiantes han destacado que el iPad ha potenciado sus habilidades expositivas y sus capacidades como profesionales de la Comunicación. Por otro lado, los profesores han visto cómo han reducido su brecha tecnológica con los alumnos gracias a la integración de esta nueva metodología docente.

Por otra parte, concluimos que el impulso de las nuevas tecnologías a través de ayudas es clave para incrementar notablemente la penetración de las TIC en las aulas (ocho de cada diez alumnos con iPad lo obtuvieron con subvención). Algo en lo que incide la Comisión Europea (2013) ante el estancamiento de las TIC que sufren las aulas de la Unión Europea.

Concluimos también que el iPad es una herramienta con un perfil más académico que los teléfonos inteligentes. Y que la integración de las tabletas digitales en el aula es tan alta que los alumnos no comprenderían su prohibición en clase después de la experiencia del proyecto piloto. No obstante, queda abierto el debate sobre la posible utilización de los teléfonos inteligentes en el aula ya que son auténticos microordenadores y cada vez lo tienen más estudiantes. El problema es que los profesores se enfrentarían a un uso poco académico de las TIC en el aula.

En lo que respecta al uso de los estudiantes de sus dispositivos vemos que los utilizan mayoritariamente para completar las explicaciones del profesor y tomar apuntes. Pero la llegada de las tabletas digitales hace que los profesores se enfrenten a nuevas situaciones. Los alumnos ya no toman solo las notas con los teclados digitales sino que también hacen uso de la cámara fotográfica para capturar los apuntes expuestos en el proyector. ¿Es tolerable este comportamiento en el aula? Quizás es algo invasivo, pero ha sido tolerado por el docente en este caso.

Por último, también llegamos a la conclusión de que el enfoque que el profesor da a la asignatura influye directamente en las aplicaciones más descargadas en clase con fines educativos. Por eso, las aplicaciones relacionadas con las exposiciones y con la práctica de Youtube fueron las más descargadas. De esta manera, la guía docente marca el uso de las herramientas tecnológicas en el aula.

\section{Referencias bibliográficas}

AREA, M. (2009). "El proceso de integración y uso pedagógico de las TIC en los centros educativos: Un estudio de casos". En: Revista de Educación, no 352, p. 77-97. Disponible en: http://redined.mecd.gob.es/xmlui/bitstream/handle/11162/ 79368/00820103009645.pdf?sequence=1. [18-09-2013].

BARTOLOMÉ, A.; AIELLO, Martín (2006). "Nuevas tecnologías y necesidades formativas: Blended learning y nuevos perfiles en Comunicación Audiovisual". En: Telos: Cuadernos de Comunicación e Innovación, nº 67, p. 59-67. Disponible 
en: http://sociedadinformacion.fundacion.telefonica.com/telos/articulocuaderno. asp@idarticulo=2\&rev=67.htm\#n12 [02-09-2013].

ESTEVE, F.; SANDOVAL, M. (2006). "Luces y sombras de las nuevas tecnologías de la información". En: Ámbitos: Revista Internacional de Comunicación, $\mathrm{n}^{\circ} 15$, p. 33-44. Disponible en: http://grupo.us.es/grehcco/ambitos\%2015/15esteve.pdf. [10-09-2013].

EUROPEAN COMMISSION (2013). "Survey of Schools: ICT in Education: Bencmarking Access, Use and Attitudes to Technology in Europe's School", February 2013. Disponible en: http://ec.europa.eu/digital-agenda/en/news/survey-schoolsict-education [23-09-2013].

GARRIDO, J. (et al.) (2008). "La Brecha de Pertinencia y el uso de recursos digitales en educación”. En: Revista Iberoamericana de Educación, n ${ }^{\circ} 3$, p. 1-11. Disponible en: http://www.rieoei.org/deloslectores/2672Miranda-Maq.pdf [12-09-2013].

GUERRA, V. (2001). "El uso de la tecnología en la educación". En: Red digital: Revista de Tecnologías de la Información y Comunicación Educativas, n ${ }^{\circ} 2$. Disponible en: http://dialnet.unirioja.es/servlet/articulo?codigo=1232512. [26-092013].

IAB (2012). "IV Estudio IAB Spain sobre Mobile Marketing: Informe de resultados", septiembre de 2012. Disponible en: http://www.iabspain.net/wp-content/uploads/ downloads/2012/09/IV-Estudio-IAB-Spain-sobre-Mobile-Marketing-Versi\%C3\%B3n-Completa.pdf. [29-09-2013].

(2013). "V Estudio Anual IAB Spain Mobile Marketing: informe de resultados", septiembre de 2013. Disponible en: http://www.iabspain.net/wp-content/uploads/ downloads/2013/09/V_Estudio_Mobile_Marketing_version_corta.pdf [29-092013].

LEÓN BARROSO, H. (2012). "De la generación del pulgar a la generación del índice". En: Teknokultura: Revista de Cultura Digital y Movimientos Sociales, vol. $9, \mathrm{n}^{\circ}$ 1, p. 177-181. Disponible en: http://teknokultura.com/index.php/tk/article/view/35 [03-10-2013].

PALMA-NIETO, A. M. (2012). "La educación en la punta de los dedos: el iPad como herramienta de motivación para el aprendizaje del vocabulario en LE". (trabajo fin de master). La Rioja: UNIR. Disponible en: http://reunir.unir.net/ handle/123456789/1513 [02-09-2013].

PÉREZ, M. J.; CABEZUELO, F. (2011). "Alfabetización y competencias digitales en los estudios de Periodismo ante el reto del Proceso de Bolonia". En: III Congreso Internacional Latina de Comunicación Social, Universidad de La Laguna, diciembre 2011. Disponible en: http://www.revistalatinacs.org/11SLCS/ actas_2011_IIICILCS/182.pdf [03-09-2013].

SÁNCHEZ, J. L. .; SORO, P. (2012). "La magia de aprender con los dedos". En: Eufonía: Didáctica de la Música, ${ }^{\circ}$ 56, Octubre - Diciembre. Disponible en: http:// eufonia.grao.com/revistas/eufonia/056-educacion-musical-y-dispositivos-moviles/la-magia-de-aprender-con-los-dedos. [14-09-2013].

SAORÍN, J. L. (et al.) (2011). "Tabletas digitales para la docencia del dibujo, diseño y artes plásticas". En: Revista TESI: Teoría de la Educación: Educación y Cultura 
en la Sociedad de la Información, $\mathrm{n}^{\circ} 12$, p. 259-279. Disponible en: http://campus. usal.es/ revistas_trabajo/index.php/revistatesi/article/view/8281/8285 [03-092013].

SIERRA, J.; CABEZUELO, F. (2010). "Recursos de las facultades de comunicación para la formación en competencias en el marco del Espacio Europeo de Educación Superior". En: Doxa Comunicación: Revista Interdisciplinar de Estudios de Comunicación y Ciencias Sociales, $\mathrm{n}^{\mathrm{o}}$ 11, p. 31-54. Disponible en: http://www. doxacomunicacion.es/pdf/artculosierraycabezuelo.pdf [19-09-2013].

SILVA OLIVARES, H.; COLLAO APABLAZA, C. (2013). "UBOPad: Uso de Tablet en apoyo a la gestión docente en el aula". En: Memorias VE2012, Panamá. Disponible en: http://reposital.cuaed.unam.mx:8080/jspui/handle/123456789/3500 [07-09-2013].

VADILLO, N.; MARTA, C. (2010). "La pizarra digital como herramienta de aprendizaje". En: Quaderns digitals: Revista de Nuevas Tecnologías y Sociedad, nº 61. Disponible en: http://dialnet.unirioja.es/servlet/articulo?codigo $=3144261$ [03-102013].

VADILLO, N.; MARTA, C.; CABRERA, D. (2010). "Proceso de adaptación de los estudios de Comunicación al EEES. El caso de Aragón, una comunidad pionera". En: Revista Latina de Comunicación Social, no 65, p. 187-193. DOI: 10.4185/ RLCS-65-2010-892-187-203. Disponible en: http://www.revistalatinacs.org/10/ art/892_Zaragoza/14_Nerea.html [23-09-2013].

VIÑAS, M.; CABEZUELO, F. (2010). "La iPad de Apple como herramienta para la formación en competencias digitales de los estudiantes de periodismo". En PÉREZ, J. M. Alfabetización mediática y culturales digitales. Sevilla. Universidad de Sevilla. Disponible en: http://www.gabinetecomunicacionyeducacion. com/files/adjuntos/LaiPaddeApplecomoherramientasparalaformacionencompetenciasdigitalesdelosestudiantesdePeriodismo.pdf [23-08-2013].

WEBB, J. (2012): The IPad as a tool for education. A case study of the introduction of IPads at Longfield Academy Kent. Disponible en: http://www.naace.co.uk/ publications/longfieldiPadresearch . [16-09-2013].

\section{El autor}

Jorge Gallardo es Doctor en Comunicación Audiovisual, Primer Premio Nacional Final de Carrera en Comunicación Audiovisual y MBA en Empresas de Televisión por la USAL. Actualmente es profesor de la Facultad de Comunicación de la Universidad Camilo José Cela donde mantiene una línea de investigación relacionada con las nuevas tecnologías y las redes sociales. Gallardo ha publicado un libro sobre Youtube y artículos en revistas de alto impacto en España vinculados al ámbito de la comunicación e Internet (Ámbitos, Zer, RLCS, Telos o Doxa). Pertenece al Grupo de Investigación en Educomunicación GIEC (S82) de la Universidad San Jorge. Además ejerce como periodista en Antena 3 donde es subdirector del programa informativo "Espejo Público". 